\title{
MORPHISMS AND INVERSE PROBLEMS FOR DARBOUX INTEGRATING FACTORS
}

\author{
JAUME LLIBRE $^{1}$, CHARA PANTAZI $^{2}$ AND SEBASTIAN WALCHER ${ }^{3}$
}

\begin{abstract}
Polynomial vector fields which admit a prescribed Darboux integrating factor are quite well-understood when the geometry of the underlying curve is nondegenerate. In the general setting morphisms of the affine plane may remove degeneracies of the curve, and thus allow more structural insight. In the present paper we establish some properties of integrating factors subjected to morphisms, and we discuss in detail one particular class of morphisms related to finite reflection groups. The results indicate that degeneracies for the underlying curve generally impose restrictions on the nontrivial vector fields which admit a given integrating factor.
\end{abstract}

\section{INTRODUCTION AND PRELIMINARIES}

This paper continues, and to some extent concludes, our work on inverse problems in the Darboux theory of integrability in the affine plane. The inverse problem for Darboux integrating factors is to characterize and determine all polynomial vector fields which admit a prescribed integrating factor. The inverse problem for invariant algebraic curves (which is a part of the former) asks for all polynomial vector fields which admit a given collection of algebraic curves as invariant sets.

Inverse problems in the Darboux theory of integrability are of interest because their solution is necessary to identify and classify the vector fields admitting a Darboux integrating factor. Moreover, such inverse problems have useful applications. For instance, Christopher [1] used an inverse problem to produce polynomial vector fields with algebraic limit cycles, and in [6] solutions of inverse problems were employed to determine vector fields of small degree with a prescribed limit cycle configuration.

In a nondegenerate geometric setting both inverse problems were essentially resolved in [2] and [3]; see also earlier work in [10], [5], [7]. Moreover, the inverse problem for curves is quite well-understood and algorithmically accessible; see [2].

The main result of [3] states that the linear space of vector fields admitting a given integrating factor is finite dimensional modulo a subspace of "trivial" vector fields, provided the underlying geometry is nondegenerate. This finiteness result was extended to arbitrary geometry in [4] with the help of sigma processes.

In the present paper we will consider general morphisms of the affine plane and discuss the behavior of invariant curves and integrating factors subject to morphisms.

1991 Mathematics Subject Classification. Primary 34C05, 34A34, 34C14.

Key words and phrases. polynomial differential system, invariant algebraic curve, Darboux integrating factor, morphism.

J.L. is supported by a MEC/FEDER grant MTM2008-03437 and by a CIRIT grant number 2005SGR 00550. C.P. is additionally partially supported by a MEC/FEDER grant MTM2006-00478. S.W. acknowledges the hospitality and support of the Mathematics Department at Universitat Autònoma de Barcelona during visits when this manuscript was prepared. 